\title{
Internal refractive index changes affect light transport in tissue
}

\author{
Ben Brooksby ${ }^{* 1}$, Hamid Dehghani ${ }^{1}$, Karthik Vishwanath ${ }^{2}$, Brian W. Pogue ${ }^{1}$, Keith D. Paulsen $^{1}$ \\ ${ }^{1}$ Thayer School of Engineering, Dartmouth College, Hanover, New Hampshire 03755 \\ ${ }^{2}$ Department of Physics and Astronomy, Dartmouth College, Hanover, New Hampshire 03755
}

\begin{abstract}
This investigation explores the effect of index of refraction, as an optical property, on light transport through optically turbid media. We describe a model of light propagation that incorporates the influence of refractive index mismatch at boundaries within a domain. We measure light transmission through turbid cylindrical phantoms with different distributions of refractive index. These distributions approximate the heterogeneous, layered nature of biological tissue. Finite element method model calculations of diffuse transmittance through these phantoms show good agreement with the trends observed experimentally. We see that phase measurements of light that propagates through approximately 90 $(\mathrm{mm})$ of scatter-dominated media may vary by 10 degrees depending upon the values of refractive index of the medium. Amplitude measurements are not as sensitive to this parameter as phase. Model calculations of diffuse reflectance from a semi-infinite slab geometry with different layers also shows good agreement with Monte Carlo simulations. We conclude that incorporating refractive index into light transport models may be worthwhile. Applying such a model in tomographic image reconstruction may improve the estimation of optical properties of biological tissues.
\end{abstract}

Keywords: Numerical modeling, near infrared tomography, boundary conditions, refractive index

\section{INTRODUCTION}

The rapidly increasing use of light in both diagnostic and therapeutic medicine has created a need to understand how radiation propagates in tissue. Mathematical models of light propagation in turbid media are required to estimate the radiation field in tissue given its optical properties and the irradiation conditions. Such "forward" calculations are necessary to predict the biological effect of therapeutic interventions such as laser surgery ${ }^{1}$ or photodynamic therapy $(\mathrm{PDT})^{2}$. The models are also used to infer the optical properties from measurements of light as it emerges from tissue. These "inverse" calculations are characteristic of diagnostic techniques such as optical tomography ${ }^{3}$ and near infrared spectroscopy (NIRS) ${ }^{4}$. Solution of the inverse problem usually involves iterative applications of a forward calculation of the light field to improve estimates of the optical properties--absorption $\left(\mu_{\mathrm{a}}\right)$ and transport scattering $\left(\mu_{\mathrm{s}}{ }^{\prime}\right)$ coefficients. Changes in optical properties can indicate physiological or pathological processes. While many homogeneous tissues can be adequately represented by a unique set of $\mu_{\mathrm{a}}$ and $\mu_{\mathrm{s}}^{\prime}$ values, imaging heterogeneous tissues may also require incorporation of a change in the index of refraction, $n$. While microscopic changes in $n$ are generally interpreted as part of the scattering coefficient, macroscopic changes in $n$ are often overlooked in NIR imaging.

Imaging of tissue with near infrared (NIR) spectral tomography is emerging as a practicable method to map hemoglobin concentration and oxygen saturation ${ }^{5-10}$. Absorption due to tissue chromophores is relatively low in this band. An optical fiber placed on the surface delivers an input optical signal (either continuous, amplitude modulated, or ultra short pulses). Other optical fibers placed at different locations on the same surface detect the exiting photons that have propagated through the volume under investigation. In the frequency domain, the amplitude and phase shift of exiting photons provide information about the optical properties of the transilluminated tissue.

Monte Carlo simulations have been found to describe the propagation of light in tissue with high accuracy ${ }^{11}$. The drawbacks of such calculations is that they are very time consuming and application of the method to arbitrary geometries with internal variations in properties is difficult to implement in an algorithm. Thus, the Monte Carlo approach has historically not been widely used in a clinical environment. It is generally agreed that the propagation of light in a strongly scattering medium such as tissue is accurately described by the equation of radiative transfer ${ }^{12}$. The

\footnotetext{
*Email: Ben.Brooksby@dartmouth.edu; Tel: (603) 646-1094; Fax (603) 646-3856
} 
radiation field at any point is characterized by the energy radiance, which is the energy transported by photons per unit time in a given direction through a unit solid angle per unit area normal to that direction. If the radiance is not strongly dependent on direction, it is possible to derive a much simpler equation for fluence rate (the number of photons passing isotropically through the surface of a unit sphere per unit time). Previous results by a number of authors show that the diffusion approximation to the radiative transfer equation is a suitable model to describe light propagation in scatterdominated materials where source and detection points are separated by at least a few scattering lengths ${ }^{13}$. A finite element method (FEM) algorithm can be implemented to provide accurate and reliable forward diffusion theory calculations for arbitrary geometries and source and detector locations ${ }^{14,15}$. This approach is further studied in this paper with the goal of adding the influence of internal changes in refractive index.

The refractive index of a material medium is the ratio of the phase speed of light in free space to that in the medium. For optical frequencies, biological tissue is reasonably considered to be a dielectric, and accordingly $n=\varepsilon_{r}^{1 / 2}$ (where $\varepsilon_{r}$ is the relative permittivity). It is known that scattering characteristics of tissues are caused by microscopic fluctuations in $n$. Refractive index is not listed in most of the literature as an optical property ${ }^{16,17}$ because it is a difficult parameter to measure in turbid media. There have been some attempts to experimentally measure $n$ of different tissues

${ }^{18-20}$, but in most numerical models index values from 1.33 to 1.6 are often assumed because the major constituent of tissue is water. While $n$ may be called a micro-index, the concept of a macro-index must be introduced to solve various boundary problems

Optical fibers transporting laser light must be placed on the surface of the tissue. Diffusion theory must account for this boundary if large errors are to be avoided in the determination of optical properties. There are three common boundary conditions applied in diffusion theory formulas (type III or partial current, extrapolated, and Dirichlet or zero) ${ }^{15}$. Type III conditions can be modified to account for Fresnel reflections that arise from the refractive index mismatch at the tissue-air interface ${ }^{21}$. Models have been developed in order to describe light propagation across other interfaces as well (i.e. tissue-tissue or tissue-fiber optic) ${ }^{22-25}$. We establish a diffusion approximation model that incorporates spatial variations in $n$ inside turbid media. We apply a modified type III condition at interior boundaries between two regions with different indices of refraction. We test this model in simulations, and perform two phantom experiments. We compare experimental results and model calculations with Monte Carlo simulations, which we consider to be accurate representations of the physical processes involved.

\section{THEORY}

Under the assumption that scattering dominates absorption in a region of interest, the Boltzmann transport equation can be simplified to the diffusion approximation. In the frequency domain this is given by:

$$
-\nabla \cdot D \nabla \Phi(\mathbf{r}, \omega)+\left(\mu_{a}+\frac{i \omega}{c}\right) \Phi(\mathbf{r}, \omega)=S(\mathbf{r}, \omega)
$$

where $S(\mathbf{r}, \omega)$ is an isotropic light source, $\Phi(\mathbf{r}, \omega)$ is the photon density at position $\mathbf{r}, c$ is the speed of light in tissue, $\omega$ is the frequency of light modulation, $\mu_{\mathrm{a}}$ is the absorption coefficient, and $D=\frac{1}{3\left(\mu_{a}+\mu_{s}^{\prime}\right)}$ is the diffusion coefficient.

The best description of the air-tissue boundary is derived with an index-mismatched type III condition, in which the fluence at the edge of the tissue exits and does not return. The flux leaving the boundary is equal to the fluence rate at the boundary, times a factor that accounts for the internal reflection of light back into the tissue. This relationship is described in the following equation ${ }^{12,14,21,26-29}$ :

$$
\Phi(\xi, \omega)=2 A \hat{\mathbf{n}} \cdot D \nabla \Phi(\xi, \omega)
$$

where $\xi$ is a point on the boundary, and $A$ depends upon the relative refractive index mismatch between tissue and air. $A$ can be derived from Fresnel's law ${ }^{23}$ :

$$
A=\frac{2 /\left(1-R_{0}\right)-1+\left|\cos \theta_{c}\right|^{3}}{1-\left|\cos \theta_{c}\right|^{2}}
$$


where $\theta_{c}=\arcsin \left(n_{2} / n_{1}\right)$, the angle at which total internal reflection occurs for photons moving from a region with $n_{1}$ to a region with $n_{2}$, and $R_{0}=\frac{\left(n_{1} / n_{2}-1\right)^{2}}{\left(n_{1} / n+1\right)^{2}}$. At external boundaries, $n_{2}=1$, the index of refraction of free space.

In finite element formalism, $\Phi(\mathbf{r}, \omega)$ is discretized into a set of basis functions multiplied by weighting coefficients, $\Phi=\sum_{j=1}^{N} \Phi_{j} \varphi_{j}$, which are determined as part of the solution process. In the Galerkin method of weighted residuals, Eq. (1) is multiplied by an identical set of weighting functions, $\varphi_{i}$, and it is integrated over the entire problem domain. After further manipulation through Green's theorem we reach Galerkin's weak form ${ }^{14,30}$ :

$$
\left\langle D \nabla \Phi \cdot \nabla \varphi_{i}\right\rangle-\oint \hat{\mathbf{n}} \cdot D \nabla \Phi \varphi_{i} d s+\left\langle\left(\mu_{a}+\frac{i \omega}{c}\right) \Phi \varphi_{i}\right\rangle=\left\langle S \varphi_{i}\right\rangle .
$$

The integrand of the surface integral is the normal component of the flux through the boundary surface. This term does not contribute to matrix assembly at node points internal to the finite element mesh boundary because of flux continuity. At interior nodes which lie on an interface between two media with different indices of refraction, we apply the conditions used by Schmitt et al and Takatani and Graham ${ }^{22,25}$.

$$
\begin{gathered}
\hat{\mathbf{n}} \cdot D_{1} \nabla \Phi_{1}(\xi, \omega)=\hat{\mathbf{n}} \cdot D_{2} \nabla \Phi_{2}(\xi, \omega) \\
\frac{\Phi_{1}(\xi, \omega)}{\Phi_{2}(\xi, \omega)}=\left(\frac{n_{1}}{n_{2}}\right)^{2}
\end{gathered}
$$

Equation (5a) ensures that the flux across a boundary remains continuous (so the flux integral in (4) still does not contribute at internal nodes), while equation (5b) establishes a discontinuity in the fluence based upon the two refractive indices defining the interface.

Implementation of $(5 b)$ in (4) occurs by separating the contributions from the domain integrations in the Galerkin weak form during matrix assembly according to the side of the interface a node belongs at an index of refraction mismatch. A simple vehicle for accomplishing this task is to duplicate nodes on the interfaced such that they possess coincident coordinate positions but unique node numbers (and therefore unique columns in the global system of algebraic equations when serving as basis functions that define the numerical photon density solutions). This allows $\Phi_{1}$ and $\Phi_{2}$ to simultaneously exist in the list of unknowns at the index-mismatched interface. On matrix assembly, one of the node numbers is preselected to act as the weighting function, and therefore, provide the row location for the discretized version of (4) on the interface. The row number associated with the partnered node is initially empty and to close the algebraic system, it is ready to accept explicit enforcement of (5b) rewritten as the equation

$$
\Phi_{1}-\left(\frac{n_{1}}{n_{2}}\right)^{2} \Phi_{2}=0
$$

\section{METHODS AND RESULTS}

Two experiments were performed, which consisted of collecting data using a near infrared tomography imaging system 31. This system is designed to image breast tissue using light with wavelengths of $660-860(\mathrm{~nm})$. It is well characterized and currently being used in a clinical trial for the diagnosis of breast disease. Sixteen evenly spaced fibers contact the surface of the pendant breast. One fiber acts as the source and the other fifteen as detectors. Here, 785 (nm) light transmission data was collected using two cylindrical phantoms with known homogeneous optical coefficients $\mu_{\mathrm{a}}$ and $\mu_{\mathrm{s}}^{\prime}$. The phantoms are made using a mixture of resin, ink (for absorption), and $\mathrm{TiO}_{2}$ (for scattering).

In the first experiment we began by measuring transmission through a solid cylinder $\left(\mu_{\mathrm{a}}=0.0055\left(\mathrm{~mm}^{-1}\right)\right.$ and $\left.\mu_{\mathrm{s}}^{\prime}=0.871\left(\mathrm{~mm}^{-1}\right)\right)$ with a diameter of $85(\mathrm{~mm})$. We then drilled a large hole through its center $(\mathrm{d}=66(\mathrm{~mm}))$, filled it with a mixture of water and intralipid with the same $\mu_{\mathrm{a}}$ and $\mu_{\mathrm{s}}^{\prime}$ as the plastic, and measured again. We used the imaging system to confirm differences in $\mu_{\mathrm{a}}$ and $\mu_{\mathrm{s}}^{\prime}$ for the two materials of less than $1 \%$. The resin is transparent, and we measured its refractive index by observing the angle by which it deflects a collimated beam. We assume this value $(n=1.58)$ is approximately equal to $n$ of the solid phantom material, and we take $n$ of the intralipid mixture as that of 
water $(n=1.33)$. Assuming no change in absorption or scattering of the medium, we look to relate the changes in the measurements to the change in $n$ within the phantom.

In Figure 1, the solid line connecting stars indicates amplitude and phase measurements (for one source and fifteen detectors, where detector \#8 is located on the opposite side of the cylinder) acquired when cylinder \#1 existed as a solid homogeneous object. The dashed line connecting stars represents measurements recorded after the phantom was drilled into a hollow cup and filled with intralipid. The data suggests that decreasing $n$ in the center of the medium causes amplitude at detector \#8 to decrease slightly. Decreasing $n$ in the center has a greater effect on the phase, causing a decrease of nearly ten degrees at detector \#8.
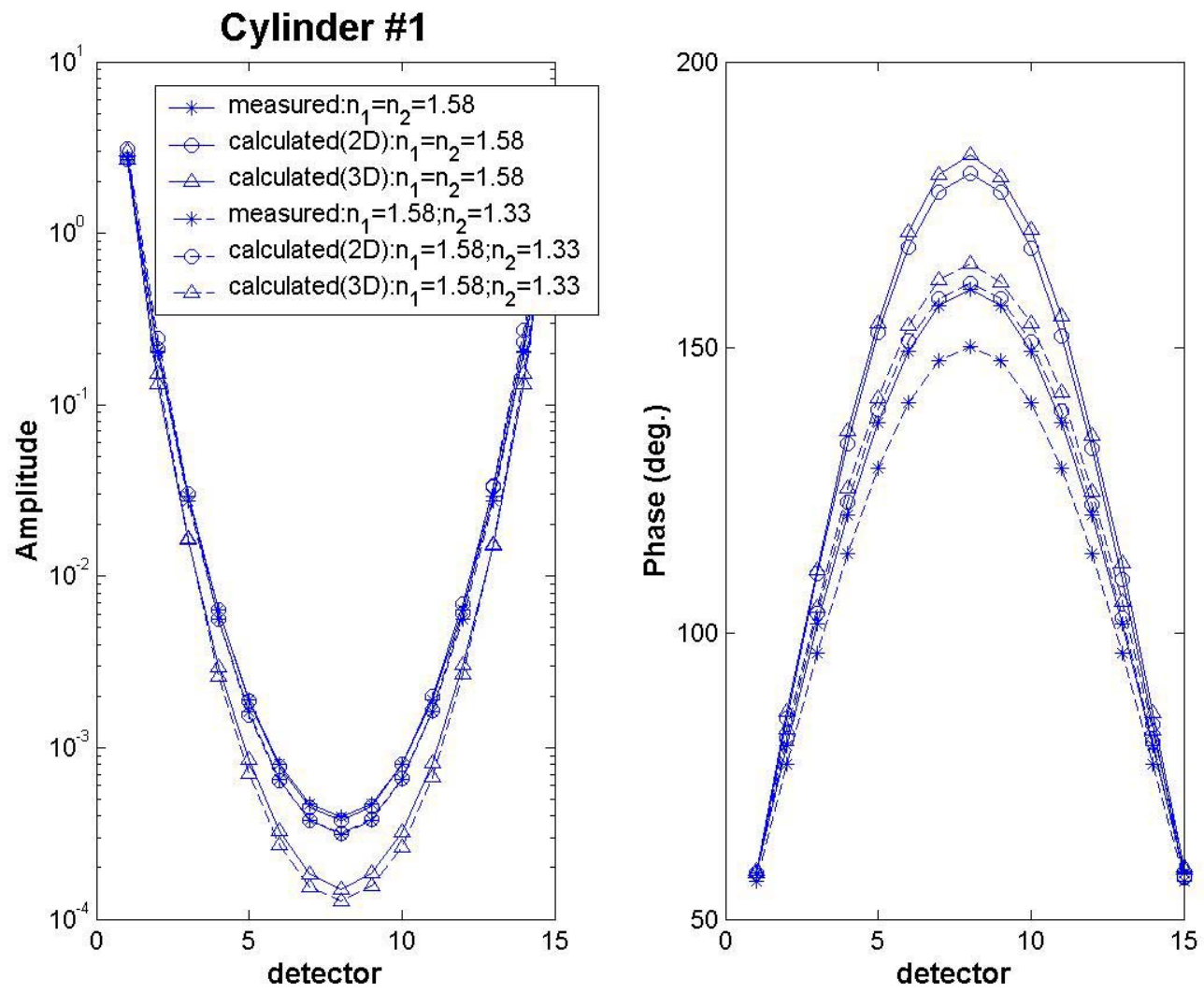

Fig. 1. Measured and simulated data from Cylinder \#1. $n_{1}$ and $n_{2}$ indicate the values in the exterior and interior of the medium, respectively. Solid lines mark data recorded from phantom with a single $n$, and dashed lines mark data acquired from the phantom after its interior was replaced with a lower value.

NIRFAST ${ }^{14}$ (Near Infrared Frequency-domain Absorption and Scatter Tomography), an algorithm for numerical modeling of light transport and image reconstruction, was used to solve the diffusion equation (Eq. 1), subject to the boundary conditions (Eq. 2 and Eq. 5). The solid and dashed lines connecting circles represent the two dimensional (2D) model calculations while the solid and dashed lines connecting triangles represent the three dimensional (3D) model computations. Both models also show slight decreases in amplitude and a decrease in phase of approximately 17 degrees when the $n$ in the center of the phantom is reduced. In Figure 1, the calculated data are normalized to the values measured at detector number one.

The same procedures were followed with a second phantom to investigate a different change in $n$. In this case the homogeneous reference was a cylinder filled with intralipid solution $\left(\mu_{\mathrm{a}}=0.0164\left(\mathrm{~mm}^{-1}\right), \mu_{\mathrm{s}}^{\prime}=0.33\left(\mathrm{~mm}^{-1}\right), n=1.33\right.$, $\mathrm{d}=92(\mathrm{~mm}))$, and the mismatch was created with a solid inclusion in the interior $(n=1.58, \mathrm{~d}=73(\mathrm{~mm}))$ having the same absorption and scattering properties. The results from this experiment are shown in Figure 2 . With the inclusion of a higher $n$, we measured a 4 degree increase in phase, and calculated a 7 degree increase in phase with both the 2D and 3D 
models. We found virtually no change in the measurements, or in the model calculations of amplitude before and after the change in $n$.
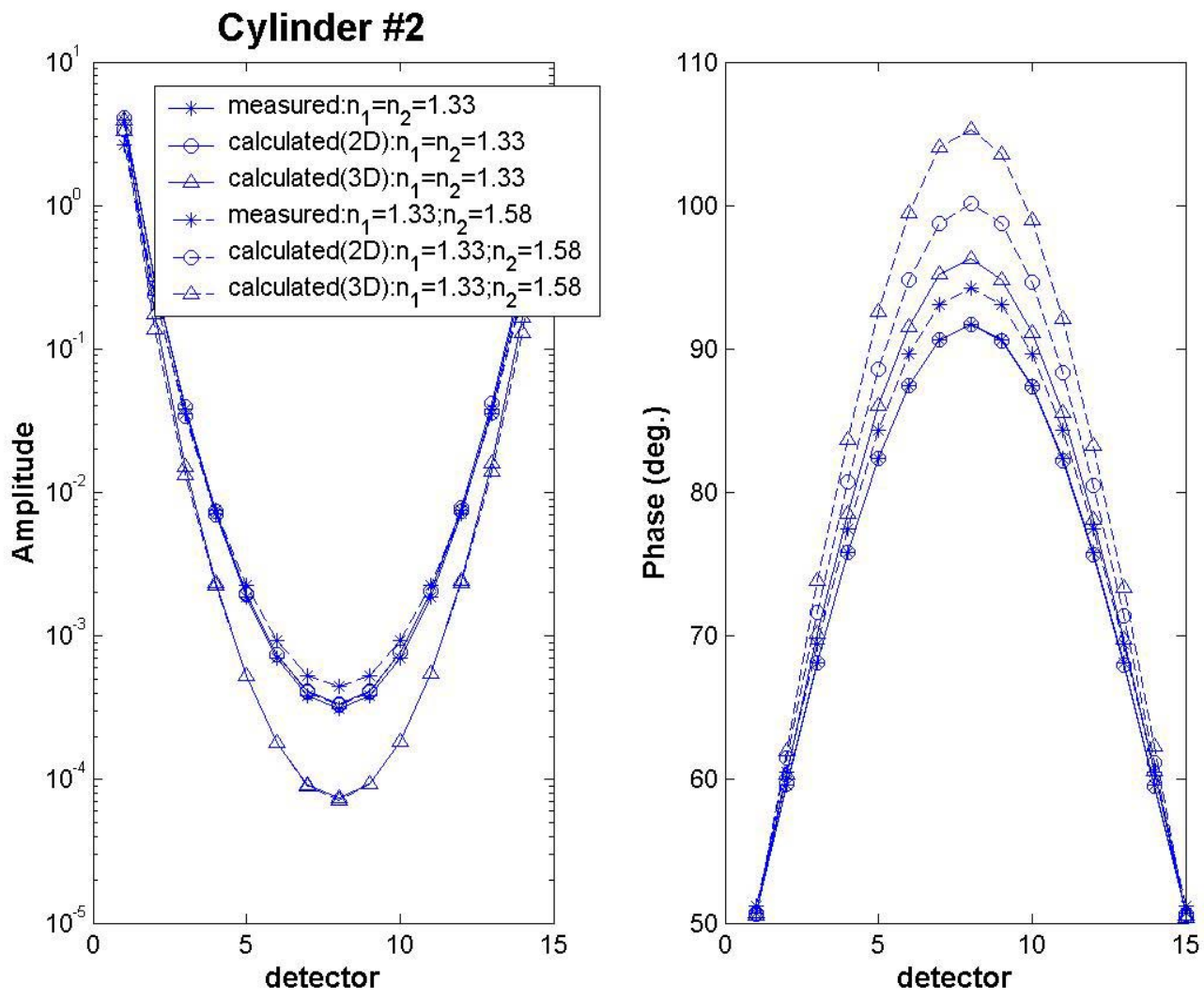

Fig. 2. Measured and simulated data from Cylinder \#2. Solid lines mark data taken from the phantom with a single $n$, and dashed lines mark data taken from the phantom after its interior was replaced with a higher $n$. With the change, there is very little effect on amplitude, and a more substantial increase in phase.

To further test the validity of our model, we compared it with Monte Carlo simulations of diffuse reflectance from a semi-infinite slab geometry. The Monte Carlo code had been validated for fluorescence lifetime spectroscopy ${ }^{32}$. We performed calculations using a slab with a thin top layer $(5(\mathrm{~mm}))$ and a much larger (semi-infinite) bottom layer. We allow for different combinations of $n$ in the different layers. A point source of photons was located in the center of the top surface and detectors measure photons exiting the slab at even distance intervals away from the source. Figure 3 shows the amplitude and phase data from simulations when the refractive index in both layers is equal $\left(n_{1}=n_{2}=1.33\right)$, and when it increases in the bottom layer $\left(n_{1}=1.33 ; n_{2}=1.58\right)$. FEM and Monte Carlo calculations are in good agreement, and show comparable phase increases at distances greater than $10(\mathrm{~mm})$ away from the source when the change is introduced. There is also a minimal decrease in amplitude at distances of 5-30 (mm). Figure 4 shows the case where both layers have the same refractive index $\left(n_{1}=n_{2}=1.58\right)$, and when it decreases in the bottom layer $\left(n_{1}=1.58 ; n_{2}=1.33\right)$. Both models show decreases in phase at distances greater than $15(\mathrm{~mm})$, and slight increases in amplitude 10-30 $(\mathrm{mm})$ from the source. 

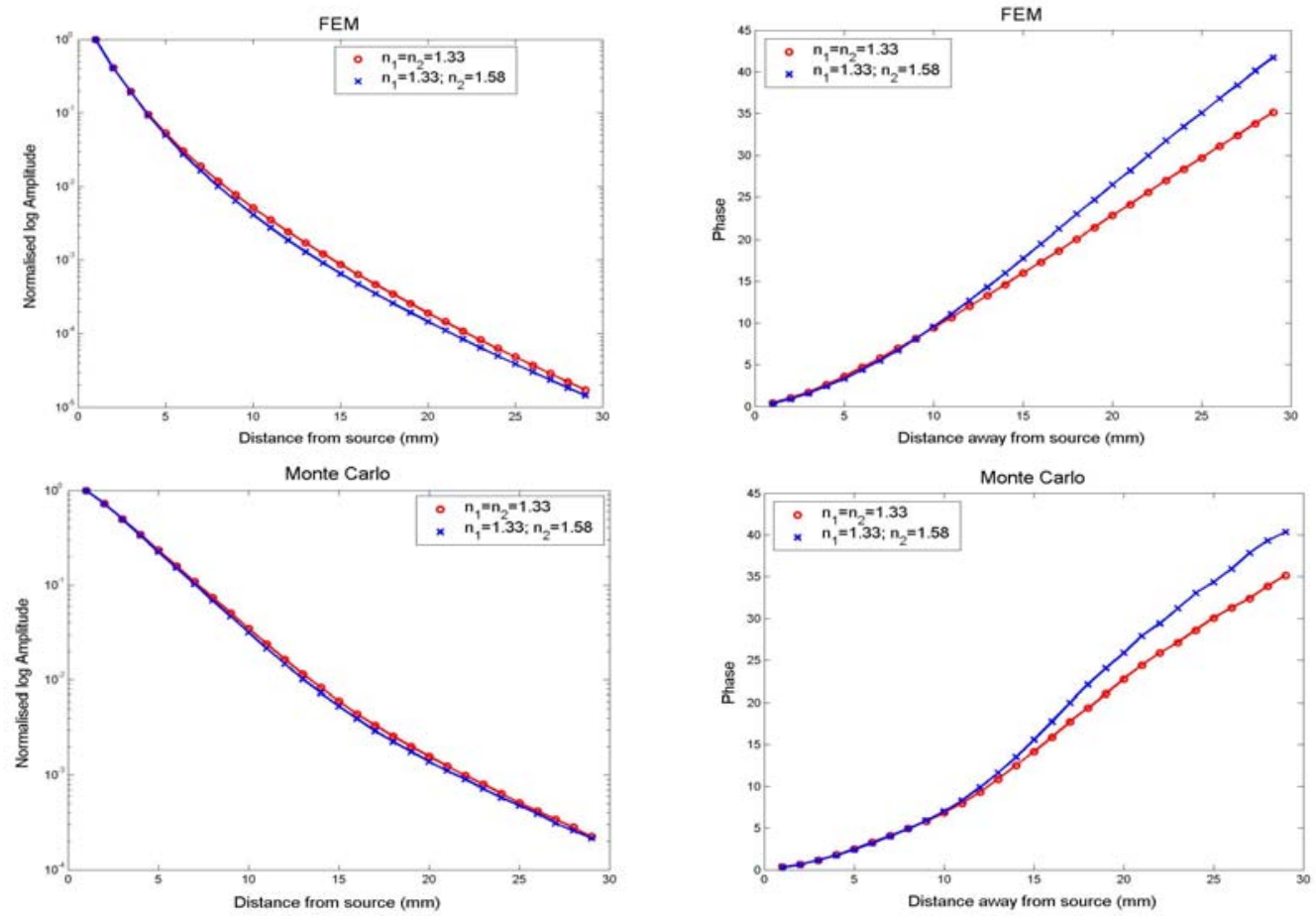

Fig. 3. Finite element method (FEM) (top two graphs) and Monte Carlo (bottom two graphs) calculations of diffuse reflectance from a semi-infinite slab geometry with layers are shown. Amplitude (left) and phase shift (right) are plotted vs. distance from the source in both cases. The two models show qualitative agreement when refractive index in the lower region of the slab increases.
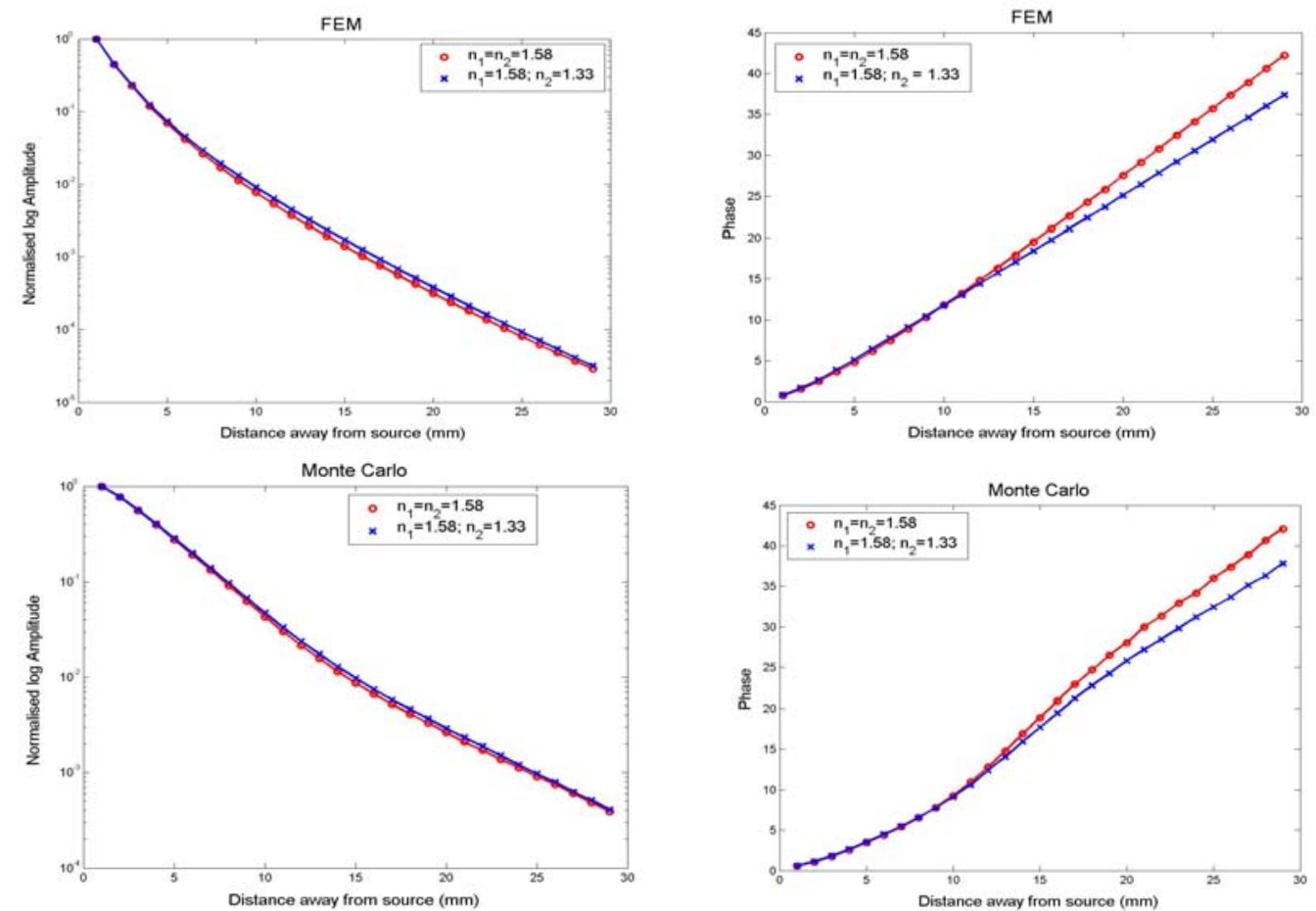

Fig. 4. Graphs of calculated amplitude (left) and phase shift (right) are shown for a thick flat slab with homogeneous refractive index, and then when the refractive index decreases in the lower region. Again, Finite element method (FEM) calculations are shown in the top two graphs, and Monte Carlo data are shown in the bottom two plots. 
We hypothesize that a model which accurately accounts for heterogeneities in index of refraction will improve biological tissue absorption $\left(\mu_{\mathrm{a}}\right)$ and scattering $\left(\mu_{\mathrm{s}}^{\prime}\right)$ image reconstructions. Figure 5 (a) shows a possible distribution of optical properties $\mu_{\mathrm{a}}, \mu_{\mathrm{s}}^{\prime}$, and $n$. We calculate light transmission through this circular geometry, and then attempt to reconstruct 2D images of $\mu_{\mathrm{a}}, \mu_{\mathrm{s}}^{\prime}$. Figure 5 (b) shows the reconstructions when a model was used that did not account for internal boundary reflections due to differences in $n$. When we use the model that does allow for these reflections, we obtain the images in Figure 5 (c). These images recover both the absorbing and scattering anomalies with better quantitative accuracy, and contain fewer artifacts. The improvements due to the $n$-sensitive model are evident in the cross sections of these reconstructions (Figure 5 (d)).

(a)

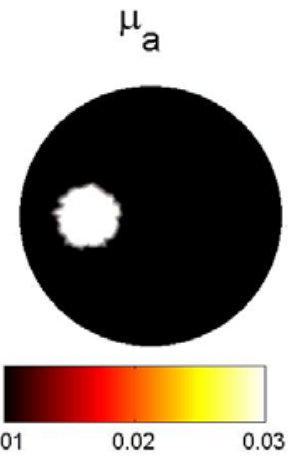

absorption coefficient
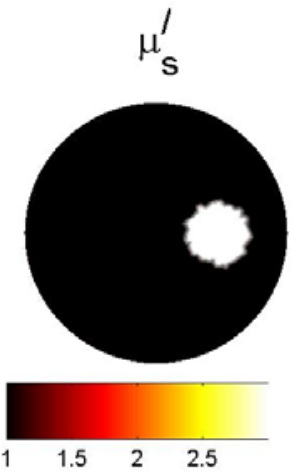

scattering coefficient
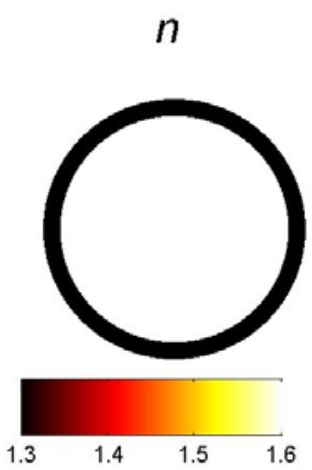

index of refraction (b)
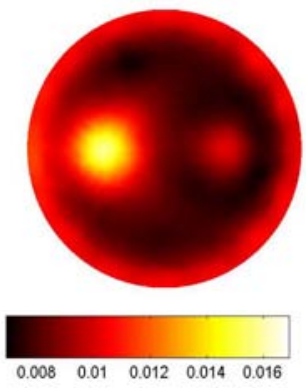
absorption coefficient
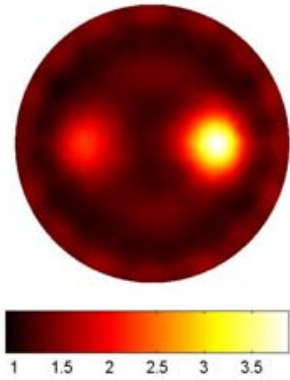

scattering coefficient $\mu_{\mathrm{a}}$

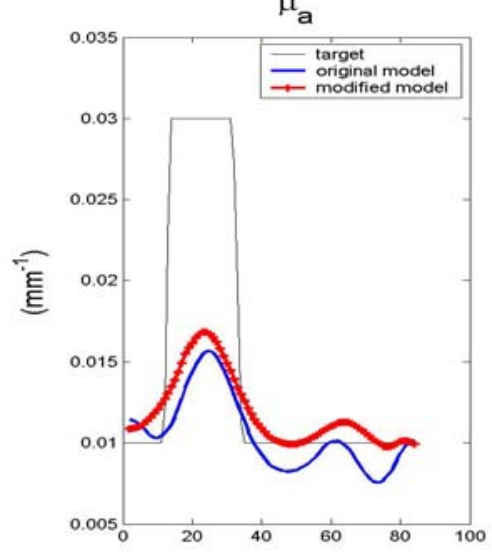

(c)
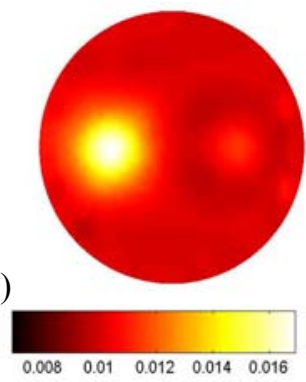

absorption coefficient
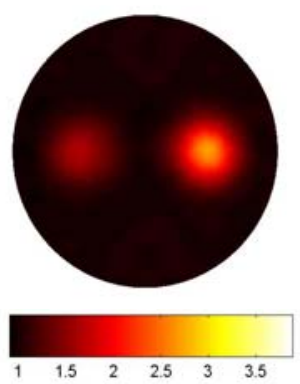

scattering coefficient $\mu_{s}^{\prime}$

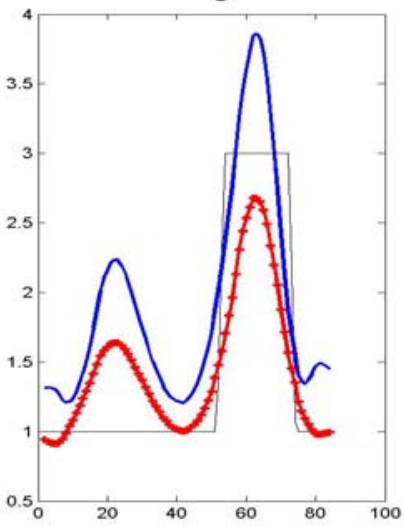

Fig. 5. (a) The target optical properties used in forward simulations are shown, including absorption coefficient, scattering coefficient and index of refraction. In this case, the effect of the outer ring of different index of refraction upon the image reconstruction algorithm is tested. Reconstructions of property distributions shown in (a) using a model which is insensitive to the refractive index change is presented in (b). Reconstruction using an algorithm that incorporated the change in refractive index a priori is shown in (c). Cross sections of the properties reconstructed in (b) and (c) are shown in (d). 


\section{DISCUSSIONS AND CONCLUSION}

The FEM model calculations do not show exact quantitative agreement with the experiments reported here (Figures 1 and 2), or the Monte Carlo simulations (Figures 3 and 4), but do represent good qualitative agreement. This work is a first attempt at incorporating multiple refractive indices into our model of light propagation through turbid media. Experimental measurements of diffuse light transmission appear sensitive to heterogeneities in refractive index. For example, in the circular array of sources and detectors that we use in tomographic imaging we see that an increase in $n$ in the center of the phantom volume causes an increase in phase and minimal change in amplitude measured at distant detectors. This result is logical considering that wavelength is reduced when $n$ is higher, and more reflection occurs when photons pass into regions with lower $n$ (based upon Fresnel's law). The increase in $n$ in the center should not directly affect how many photons are absorbed, but the photon pathlength should increase. This phantom could represent the heterogeneous refractive index distribution of breast tissue. Adipose and glandular tissues have different compositions.

More experiments are necessary to confirm the consistency of this model and experimental observations. Our model in both 2D and 3D shows the same trends in amplitude and phase as Monte Carlo simulations. In the cylindrical imaging geometry, the model calculations of phase sensitivity to $n$ slightly overestimate what we observed with our imaging system. The model calculations and measurements of amplitude reveal almost no variation. In the future we plan further development of this model. Refractive index clearly has an effect upon measurements in diffuse optical

tomography, but it is a difficult parameter to measure independently. Experiments are required that will elucidate this affect.

\section{ACKNOWLEDGEMENTS}

Funding for this work has been provided from National Cancer Institute through research grants PO1CA80139 and RO1CA69544.

\section{REFERENCES}

$1 \quad$ Boulnois, J.-L.,Photophysical processes in recent medical laser developments: A review Lasers in Medical Science 1, 47 (1986).

2 Johansson, T., Thompson, M. S., Stenberg, M., Klinteberg, C., Andersson-Engels, S., Svanberg, S., and Svanberg, K.,Feasibility study of a system for combined light dosimetry and interstitial photodynamic treatment of massive tumors Applied Optics 41, 1462 (2002).

3 Arridge, S. R.,Optical tomography in medical imaging Inverse Problems 15, R41 (1999).

4 Arridge, S. R., van der Zee, P., Cope, M., Delpy, D. T.,Reconstruction methods for infrared absorption imaging Proc. SPIE 1431, 204 (1991).

5 Pogue, B. W., Zhu, H., Nwaigwe, C., McBride, T. O., Osterberg, U. L., Paulsen, K. D., Dunn, J. F.,Hemoglobin Imaging With Hybrid Magnetic Resonance and Near-Infrared Diffuse Tomography Adv,. Expt. Med. Biol., (in press) (2000).

$6 \quad$ Jiang, S., Pogue, B. W., McBride, T. O., and Paulsen, K. D.,Quantitative Analysis of Near-Infrared Tomography: Sensitivity to the Tissue-Simulating Pre-Calibration Phantom J. Biomed. Opt. (in press) (2002). Hebden, J. C., Veenstra, G., Dehghani, H., Hillman, E. M. C., Schweiger, M., Arridge, S. R., and Delpy, D. T.,Three dimensional time-resolved optical tomography of a conical breast phantom Applied Optics 40, 3278 (2001).

$8 \quad$ Fantini, S., Schutz, O., Edler, J., Franceschini, M. A., Heywang-Krbrunner, S., Gotz, L., Siebold, H., in SPIE Technical Abstract Digest, edited by B. Chance (SPIE Press, San Jose, CA, 1999), p. 103.

9 Bremer, C., Ntziachristos, V., Mahmood, U., Tung, C. H., and Weissleder, R.,[Progress in optical imaging] Radiologe 41, 131 (2001). 
Boas, D., Gaudette, T., Arridge, S.,Simultaneous imaging and optode calibration with diffuse optical tomography Opt. Exp. 8, 263 (2001).

Flock, S. T., Wilson, B. C., and Patterson, M. S.,Monte Carlo modeling of light propagation in highly scattering tissues--II: comparison with measurements on phantoms IEEE Trans. Biomed. Eng BME-36, 1169 (1992).

Patterson, M. S., Moulton, J. D., Wilson, B. C., Berndt, K. W., Lakowicz, J. R.,Frequency-domain reflectance for the determination of the scattering and absorption properties of tissue Appl. Opt. 30, 4474 (1991). Dehghani, H., Pogue, B. W., Poplack, S. P., and Paulsen, K. D.,Multiwavelength Three-Dimensional NearInfrared Tomography of the Breast: Initial Simulation, Phantom, and Clinical Results Appl. Opt. 42, 135 (2003).

Dehghani, H., Pogue, B. W., and Paulsen, K. D., in OSA Biomedical Topical Meetings, Miami Beach, Florida, 2001), Vol. 2002 Technical Digest.

Jiang, H., Paulsen, K. D., in Proc. SPIE: Optical Tomography, Photon migration and spectroscopy of tissue and model media, edited by B. Chance, Alfano, R. R., San Jose, CA, 1995), Vol. 2389, p. 608.

Cheong, W., Prahl, S. A., and Welch, A. J.,A review of the optical properties of biological tissues IEEE journal of quantum electronics 26, 2166 (1990).

Durduran, T., Choe, R., Culver, J. P., Zubkov, L., Holboke, M. J., Giammarco, J., Chance, B., and Yodh, A. G.,Bulk optical properties of healthy female breast tissue Phys. Med. Biol. 47, 2847 (2002).

$\mathrm{Li}, \mathrm{H}$. and Xie, S.,Measurement method of the refractive index of biotissue by total internal reflection Applied Optics 35, 1793 (1996).

Bolin, F. P., Preuss, L. E., Taylor, R. C., and Ference, R. J.,Refractive index of some mammalian tissues using a fiber optic cladding method Appl. Opt. 28, 2297 (1989).

Bartlett, M. A. and Jiang, H.,Effect of refractive index on the measurement of optical properties in turbid media Appl. Opt. 40, 1735 (2001).

Arridge, S. R., Schweiger, M.,Sensitivity to prior knowledge in optical tomographic resconstruction Proc. SPIE 2389, 378 (1995).

Takatani, S. and Graham, M.,Theoretical analysis of diffuse reflectance from a two-layer tissue model IEEE Trans. Biomed. Eng 26, 656 (1979).

Keijzer, M., Star, W. M., and Storchi, P. R. M.,Optical diffusion in layered media Appl. Opt. 27, 1820 (1988). Kienle, A., Patterson, M. S., Dognitz, N., Bays, R., Wagnieres, G., and van den Bergh, H.,Noninvasive determination of the optical properties of two-layered turbid media Appl. Opt. 37, 779 (1998).

Schmitt, J. M., Zhou, G. X., Walker, E. C., and Wall, R. T.,Multilayer model of photon diffusion in skin J. Opt. Soc. Am A 7, 2141 (1990).

Farrell, T. J., Patterson, M. S., and Wilson, B. C.,A diffusion theory model of spatially resolved, steady-state diffuse reflectance for the noninvasive determination of tissue optical properties in vivo Medical Physics 19, 879 (1992).

Groenhuis, R. A. J., Ferwerda, H. A., ten Bosch, J. J.,Scattering and absorption of turbid materials determined from reflection measurements. I.Theory. Appl. Opt. 22, 2456 (1983).

Haskell, R. C., Svaasand, L. O., Tsay, T. T., Feng, T. C., McAdams, M. S., and Tromberg, B. J.,Boundary conditions for the diffusion equation in radiative transfer J. Opt. Soc. Am. A 11, 2727 (1994).

Hielscher, A. H., Jacques, S. L., Wang, L. V., and Tittel, F. K.,The influence of boundary conditions on the accuracy of diffusion theory in time-resolved reflectance spectroscopy of biological tissue Phys. Med. Biol. 40, 1957 (1995).

Lapidus, L. L. and Pinder, G. F., Numerical Solution of Partial Differential Equations in Science and Engineering (John Wiley \& Sons, Inc., New York, 1999).

McBride, T. O., Pogue, B. W., Jiang, S., Osterberg, U. L., and Paulsen, K. D.,Development and Calibration of a Parallel Modulated Near-Infrared Tomography System for Hemoglobin Imaging In Vivo Rev. Sci. Instr. 72, 1817 (2001).

Vishwanath, K., Pogue, B. W., and Mycek, M. A.,Quantitative Fluorescence Lifetime Spectroscopy in Turbid Media: Comparison of Theoretical, Experimental and Computational Methods Phys. Med. Biol. 47, 3387 (2002). 